\title{
FORMATION OF THE INTERNATIONAL CELESTIAL REFERENCE FRAME
}

\author{
C. MA \\ NASA, Goddard Space Flight Center, Greenbelt, MD 20771, USA \\ E.F. ARIAS \\ Facultad de Ciencias Astronomicas y Geofisicas de La Plata; \\ Observatorio Naval Buenos Aires; CONICET, Argentina
}

T.M. EUBANKS AND A.L. FEY

US Naval Observatory, Washington, USA

A.-M. GONTIER

Central Bureau of IERS, Observatoire de Paris

AND

C.S. JACOBS AND O.J. SOVERS

$J P L$, Pasadena, USA

\section{Introduction}

The goal of the work described here is to create the definitive catalogue for the new International Celestial Reference Frame (ICRF) using the best data and methods available at the time the work was done. This work is the joint cooperative effort of a subgroup of the IAU Working Group on Reference Frames which was formed expressly for this purpose in February 1995. The anthors of this report constituted the subgroup. A fuller account of this report can be found in the introduction to the ICRF catalog (IERS 1997).

The ICRF of 608 sources presented here is based on essentially all the VLBI observations accumulated over about 15 years in several worldwide programs. Dual frequency Mark III data have both geodetic and astrometric applications. Most of the data ( $95 \%$ of nearly 2 million observations) were acquired primarily for geodetic purposes. The major geodetic programs include: NASA's Crustal Dynamics Project/Space Geodesy Program and USNO's NAVEX sessions for the terrestrial reference frame, as well as IRIS, NAVNET and NEOS sessions for monitoring Earth rotation. The geodetic programs have used the brightest radio sources, gradually concentrating on the most compact as sensitivity improved. These geodetic sources were also the foundation of astrometric work because of the large number of observations for the $\sim 150$ most commonly used. The astrometric programs which densify the sky include the Radio-Optical Reference Frame sessions done by US Naval Research Laboratory (NRL) and USNO and the space navigation efforts of Jet Propulsion Laboratory (JPL).

The subgroup has taken an empirical approach in the selection of data, analysis, estimation of errors, and categorization of the final results. The characterization of a radio source, i.e., its position, how it was treated in the analysis, and whether it was suitable for use as a defining object, was derived entirely from the VLBI data and analysis and not from any other information. This approach leads to a rigorous selection of defining objects and a reliable realization of the ICRF as a set of relative positions oriented to the axes of the International Celestial Reference System, ICRS (Arias et al., 1995).

It was considered essential that the realization of the ICRF be derived from a single analysis, even if imperfect, rather than from a combination catalogue made of several VLBI solutions. 


\section{Preparation for analysis}

As suggested above, the sources can be characterized along several lines. The most important are variations in position seen in the data and number of observations. The underlying conceptual basis of this type of realization of the celestial reference frame is that positions are invariant with time. Therefore, the first task was to ensure that this condition was not significantly violated.

A series of solutions was made. In each solution the positions of all sources except for a small test set were estimated as time-invariant parameters. The position of each test source was re-estimated for each day the source was observed. Each source was treated as a test source in some solution. The complete set of source positions as functions of time was analysed to determine which sources had statistically significant variations in their positions. In addition, in another solution apparent linear motion was estimated simultaneously for all sources with sufficient data, generally two or more observing sessions. Two modes of variation were recognized. For sources with sufficient data to derive statistically significant apparent linear motions, a source was considered problematical if the apparent motion exceeded $50 \mu \mathrm{as} / \mathrm{yr}$ and was larger than the formal error.

Other sources were rejected if the weighted root-mean-square of the source position variations exceeded 0.5 milliarcsecond (mas) in arc length and were also more than $4 \sigma$ based on the formal position errors. Since these two classes of sources showed undesirably large position variation, they were treated differently from other sources in the final analysis. To accommodate their position variations without deforming the rigid geometry of the remaining sources, the positions of these sources were adjusted separately for each session in which they were observed.

The variations in position seen in the dataset were not comprehensively analyzed to determine the causes. The position variations for some sources show clear correlations with changes in mapped structure and to some extent the position changes can be derived from the structure, but there was no strong evidence of any regularly repeated behavior.

\section{Configuration of the ICRF analysis}

The configuration of the ICRF analysis was developed as a balance between competing goals: the most data and the least systematic error, the best models and available options, the largest number of useful estimated parameters and computer speed, and the like.

The most important choices were related to data and modeling/estimation. To extract the most information from the data set both delay and delay rates were used, and only observations below 6 degrees were excluded. The troposphere was modeled using the MTT mapping function (Herring, 1992). Time-variable troposphere gradients were estimated.

The primary geodetic parameters, the station positions, were estimated separately for each session. In this way any non-linear motion of the stations does not affect the integrity of the invariant source positions. The unadjusted a priori models for geophysical effects and precession/nutation generally followed the IERS Standards 1992 (McCarthy, 1992). The VLBI theoretical model was the so-called consensus model given in the IERS Conventions 1996 (McCarthy, 1996).

The parameters were estimated using arc-parameter elimination (Ma et al., 1990), which is an incremental least-squares method that can accommodate large numbers of parameters if they are associated only with particular data intervals. In the ICRF analysis several classes of parameters were adjusted. For each observing session, the adjusted arc parameters included:

1. positions of 'arc' sources with identified excessive apparent motion or random variation,

2. two celestial pole offsets to account for errors in the standard precession/nutation models,

3. positions of the stations,

4. the rate of UT1 relative to a good a priori time series,

5. 1-hour troposphere parameters described above,

6. troposphere gradients in the $\mathrm{E}-\mathrm{W}$ and N-S directions, linear in time,

7. quadratic clock polynomials for the gross clock behavior,

8. 1-hour clock parameters similar to the 1-hr troposphere parameters, and

9. necessary nuisance parameters such as clock jumps and baseline clock offsets.

Certain parameters were adjusted as invariant quantities from the entire dataset. These global parameters included:

1. invariant source positions, 
2. geometric axis offsets for all fixed antennas (small effect), and

3. 252 parameters for Earth rotation variations in the diurnal and semidiurnal bands caused by ocean tides (small effects).

After a series of test solutions to refine various aspects of the analysis, a final solution was run which included 1.6 million pairs of delays and rates from August 1979 through July 1995.

\section{Determination of realistic error}

Given the very large number of observations for some sources, the error contribution from their observation noise was very small and not a meaningful measure of uncertainty. It was necessary to consider several other effects to assign realistic errors. One was the statistical validity of the formal errors. Another was the cumulative influence of all modeling errors and editing decisions. Yet another was the magnitude of specific, identifiable systematic errors that could have distorted the results.

The cumulative effect of modeling errors and data editing can be examined by comparison of results from independent analyses. Intercomparison of different radio source catalogues addresses the problem of mismodeling and consequent systematic errors. Aspects that can be probed by catalogue comparisons include:

1. extension of the data set used in analysis;

2. analysis differences at different centers;

3. agreement between subsets of data;

4. intentional perturbations of modeling.

A number of such comparisons involved the ICRF, associated catalogues from the identical data base that were created for test purposes, and existing radio source catalogues. Existing catalogues included the 1994 and 1995 IERS realizations of the celestial reference frame (IERS94 and IERS95) and the radio-optical reference frame of Johnston et al. (1995).

From a consideration of all the known error sources, the subgroup concluded that a realistic error estimate for the invariant source positions could be made by inflating the formal errors by a factor of 1.5 followed by a root-sum-square increase of 0.25 mas. For the most frequently observed sources the 0.25 mas was the dominant error. The errors of the arc sources were also increased by 0.25 mas.

To address the question of whether adopting a unique 'error calibration law' for all sources climinated or at least minimized systematic effects, a combined frame was made by adopting a 6-parameter comparison model (three angles, drifts in right ascension and declination as functions of declination, and a bias in declination). Individual VLBI frames submitted by GSFC, JPL, NOAA and USNO to the IERS in 1995 (Charlot, 1995) were then included in this combination. For some sources position uncertainties obtained from the catalog combination were larger than predicted, so for these sources the uncertainty of each coordinate was set to the larger of the inflated or comparison value.

\section{Categorization of sources}

To be most useful in defining the ICRF a source should ideally show no variation in position in the dataset, have sufficient data to support the absence of variation, and not have shown unexplained differences in position between realizations of equivalent validity. Several quality levels can be established for each of the 608 sources in the ICRF catalogue. These were based on one of three sets of criteria:

1. quality of datia and observation history

2. consistency of coordinates derived from subsets of data

3. repercussions of source structure.

To qualify for the list of sources that was used to orient the ICRF with respect to the IERS celestial reference system, a source had to meet these three conditions. Some criteria that can be used to identify 'problem sources' are related to their internal structure and their variation with time. If the structure varies with time, the effective centroid can move. Even with static structure, the baselines and their time-varying orientation can induce apparent shifts in position. A simple 
way to assess the time-variability is to estimate rates of change of right ascension and declinations with time. A more direct assessment of the effects of extended source structure was provided by using externally generated source maps to calculate structure corrections to VLBI observables. A number of such maps were available from the work of Fey with the VLBA.

Sources failed to be defining sources for any of the following reasons:

1. Arclength formal error $>1$ mas

2. $<20$ observations

3. $<2-$ year span of data

4. $3 \sigma$ or $>0.5$ mas discrepancy between catalogues

5. excessive structure

6. large, significant apparent motions

7. arc source, i.e., position adjusted for each session.

The sources then fell into three categories: 212 defining sources that failed none of the criteria, 294 candidate sources that failed some or all of the criteria, and 102 sources with identified excessive position variation, either linear or random. Figure 1 shows histograms of the inflated errors of the 212 defining sources. Candidate sources potentially could be designated defining sources in future

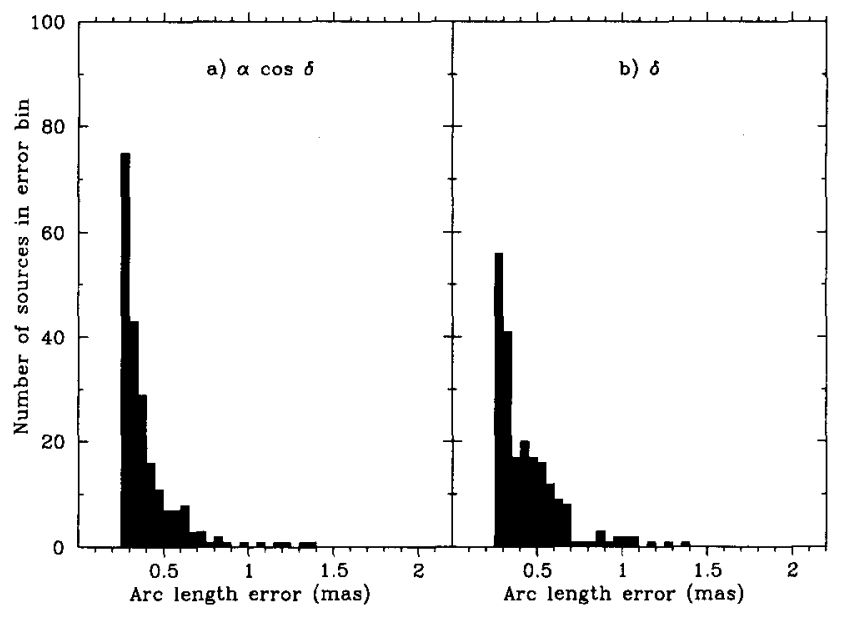

Figure 1. Histograms of position errors for defining sources.

realizations of the ICRF as more data become available or analysis improves. The third category of other sources does include sources that may be useful for purposes such as radio-optical frame ties. While only the defining sources have a formal role in the ICRF, the positions of all sources are consistent with the ICRF.

\section{Orientation of the ICRF}

The final stage in the ICRF realization was the rigid rotation of the relative positions to the ICRS maintained by the IERS. The ICRF was aligned to the ICRS by rotating it onto the last realization of the IERS celestial reference frame, RSC(IERS) $95 \mathrm{C} 02$ (IERS, 1996). Radio source coordinates in RSC(IERS) $95 \mathrm{C} 02$ were evaluated by combining individual extragalactic reference frames submitted to the IERS in 1995. The coordinates adopted for a set of 236 defining sources aligned the axes of RSC(IERS) $95 \mathrm{C} 02$ to the IERS celestial reference system.

Due to the model adopted in the compilation of RSC(IERS) $95 \mathrm{C} 02$, the frame was affected by the deformations coming from the individual catalogues. The improvements in the models and procedures applied in the ICRF solution resulted in a frame free from deformations but slightly misaligned with respect to RSC(IERS) $95 \mathrm{C} 02$. In the procedure applied to rotate the ICRF solution to IERS frame, care was taken not to transfer to the former the deformations of the latter. 


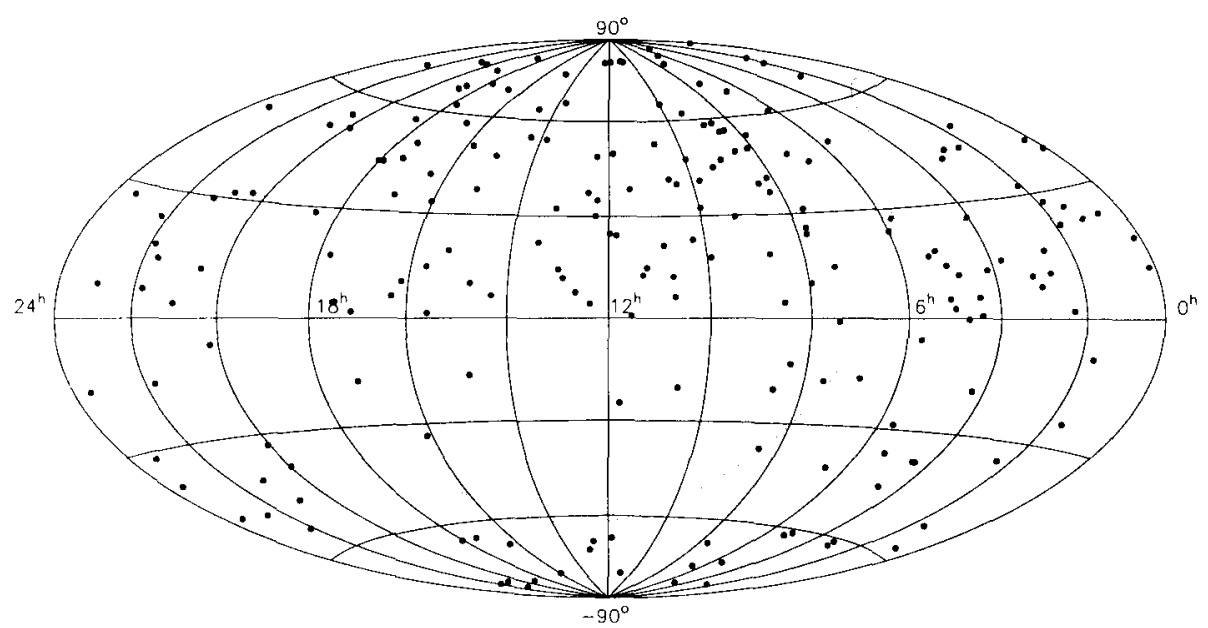

Figure 2. Areal distribution of 212 defining sources.

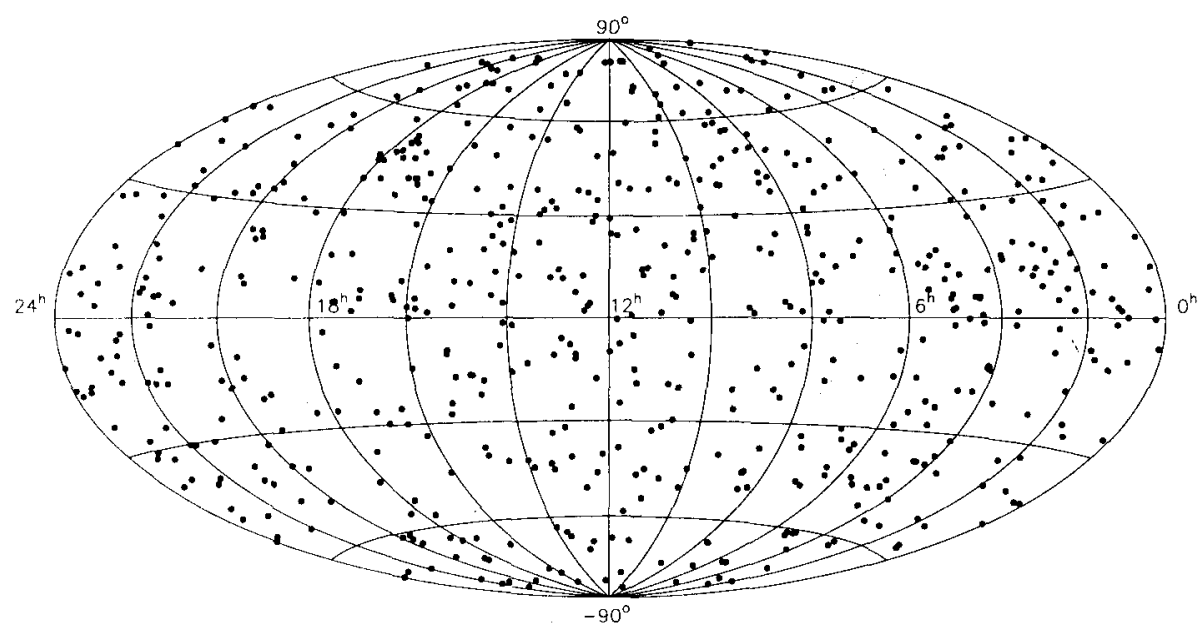

Figure 3. Areal distribution of all 608 ICRF sources.

\section{The ICRF catalogue}

Figure 2 shows the distributions of objects on the sky for the 212 defining sources, and Figure 3 for all the 608 sources. It can be seen that there is moderately even distribution of all sources, but the Southern hemisphere is deficient in defining sources. This is caused by the small number of VLBI stations in the South and limited observing programs. While all sources are given ICRF designations, it should be emphasized that the quality and intended use of the three categories are quite differcnt. The best astrometric quality resides in the defining sources and those candidate sources with the smallest errors. The positions of the 'other' sources should be used carefully and only where less accuracy can be tolerated. 


\section{Evolution of the ICRF}

The current realization condenses the information from a particular VLBI dataset spanning a defined interval and reflects a certain state of VLBI analysis. As time progresses we expect the realization of the ICRF to evolve, although changes in the ICRF catalogue will be infrequent compared to past practice in VLBI astrometry.

Current sources need to be observed periodically to track their behavior. Second, as observations accumulate, it should be possible to move candidate sources up or down the scale of usefulness. However, it is conceivable, perhaps even probable, that an identical categorization of sources from an analysis using twice as long an interval would show sources changing categories in unpredictable ways. The problem of position variation may be solved in the future if the application of source structure information permits the identification and use of truly kinematically stable points in the sky. This remains to be demonstrated. Unlike stellar catalogues, however, the original VLBI observations should always be accessible for improved analysis de novo.

Despite the burden of maintenance, the ICRF realized by VLBI astrometry is a great step forward. Compared to stellar realizations it is intrinsically simpler, much more accurate, more stable, and less susceptible to systematic deformations.

\section{References}

Arias, E.F., Charlot, P., Feissel, M., Lestrade, J.-F. (1995) The Extragalactic Reference System of the International Earth Rotation Service, ICRS, A\&A 303, 604-608

Charlot, P. (1995) IERS Technical Note No.19, Observatoire de Paris

Herring, T.A. (1992) Modeling atmospheric delays in the analysis of space geodetic data, Symposium on Refraction of Transatmospheric Signals in Geodesy [Eds. J C. De Munk \& T.A. Spoelstra], Netherlands Geod. Cornm., Delft, 157-164

IERS (1996) 1995 IERS Annual Report, Observatoire de Paris, II-19.

IERS (1997) Definition and Realization of the International Celestial Reference System by VLBI Astrometry of Extragalactic Objects, [Eds. C. Ma \& M. Feissel] IERS Technical Note No.23, Observatoire de Paris

Johnston, K.J., Fey, A.L., Zacharias, N., Russell, J.L., Ma, C., de Vegt, C., Reynolds, J.E., Jauncey, D.L., Archinal, B.A., Carter, M.S., Corbin, T.E., Eubanks, T.M., Florkowski, D.R., Hall, D.M., McCarthy, D.D., McCulloch, P.M., King, E.A., Nicolson, G., Shaffer, D.B. (1995) A radio reference frame, AJ 110, 880-915

Ma, C., Sauber, J.M., Bell, L.J., Clark, T.A., Gordon, D., Hirnwich, W.E., Ryan, J.W. (1990) Measurement of horizontal motions in Alaska using very long baseline interferometry, J. Geophys. Res. 95, 21991-22011

McCarthy, D.D. [Ed.] (1992) IERS Standards 1992, IERS Technical Note No.13, Observatoire de Paris

McCarthy, D.D. [Ed.] (1996) IERS Conventions 1996, IERS Technical Note No.21, Observatoire de Paris 